
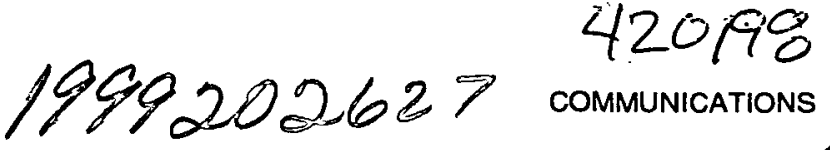

COMMUNICATIONS

This report is a preprint of an article submitted to a journal for publication. Because of changes that may be made before formal publication, this preprint is made available with the understanding author.

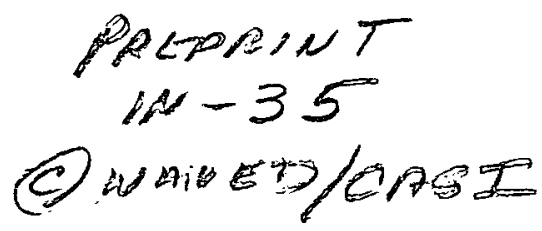

\section{Gaussian beam intensity flattener}

\author{
DeVon W. Griffin \\ NASA Lewis Research Center \\ Microgravity Fluid Physics Branch \\ M/S 100-3 \\ 21000 Brookpark Road \\ Cleveland, Ohio 44135 \\ E-mail: devon.griffin @lerc.nasa.gov
}

Subject terms: lens design; Gaussian beams.

Paper CM-2127 received Dec. 24, 1997; revised manuscript received March 14, 1998; accepted for publication March 14, 1998. [S0091-3286(98)03707-6]

\section{Introduction}

A recent article ${ }^{1}$ outlined the procedure for using a gradient index lens system to change a Gaussian intensity distribution to one that is uniform without any loss of flux to the $1 / e^{2}$ limit. While design of this system was straightforward, practical implementation has been delayed due to fabrication difficulties. ${ }^{2}$ Even though the pioneering work on such intensity transformations was performed nearly 30 years ago, ${ }^{3}$ implementation difficulties have been the genesis of discussions involving many different approaches such as aspheres, ${ }^{4}$ holograms, ${ }^{5}$ and aberrated spherical optics. ${ }^{6}$
Shafer ${ }^{6}$ discussed the use of spherical elements to generate a uniform beam to the $1 / e$ diameter. The goal of this investigation was to use commercial elements and extend the correction to a $1 / e^{2}$ diameter of $3 \mathrm{~mm}$ over long propagation distances.

\section{Design Procedure}

The starting point for this design consisted of using two doublets, as suggested by Shafer. ${ }^{6}$ Rather than use spherical aberration as a tool to redistribute the energy, as he and Frieden ${ }^{3}$ did, ray locations were used to represent energy distribution as discussed by other workers. ${ }^{1,4,5}$ In those cases, however, use of either custom gradient or aspheric optics assured perfect correction. In this investigation, neither option was used. While the net result for one of the designs presented here was excellent to the $1 / e$ point and acceptable to the $1 / e^{2}$ points, the results do suffer from the inability to independently address each zone, a capability that is only available with holograms, aspheres, or custom GRIN devices.

The rayset used for optimization was chosen by first requiring that rays represent the boundary of a region of equal energy in the Gaussian beam. This stipulation resulted in a heavier sampling of the beam near the optical axis where the irradiance was highest. It was accomplished by calculating the volume under a unity amplitude twodimensional Gaussian and dividing by 20 , the desired number of sampling locations in the merit function. A numerical integration was then iteratively performed to identify the boundaries of these regions of equal irradiance.

The targets in the merit function were the transformed locations of these rays in the output beam. The transformation assumed conservation of energy between the input Gaussian and output uniform beams. Mathematically, the result is ${ }^{5}$

$R=\left\{\frac{r_{0}^{2}}{2 \sigma}\left[1-\exp \left(-2 r^{2} / r_{0}^{2}\right)\right]\right\}^{1 / 2}$

where $r$ is the radius of the input ray, $R$ the position of the corresponding output ray, $r_{0}$ is the $1 / e^{2}$ radius of the Gaussian beam, and $\sigma$ is the uniform irradiance for correction to that radius.

Shafer ${ }^{6}$ proposed four different design forms, but only extensively investigated the $(+-,-+)$ case to the $1 / e$ radius. When this form was used with the merit function previously described and correction extended to the $1 / e^{2}$ radius, the beam remained approximately collimated between the doublets while performing the energy redistribution to what essentially was a first approximation. Though uniform illumination at a surface $50 \mathrm{~mm}$ following the last lens was achieved, that profile did not persist with increasing propagation distance. To increase the performance, both doublets were changed to triplets and the optimization continued. Once the change from the doublets had been made, the light was no longer approximately collimated in the center section. Rather, it formed a focus between the third and fourth and fourth and fifth elements. A split of the 


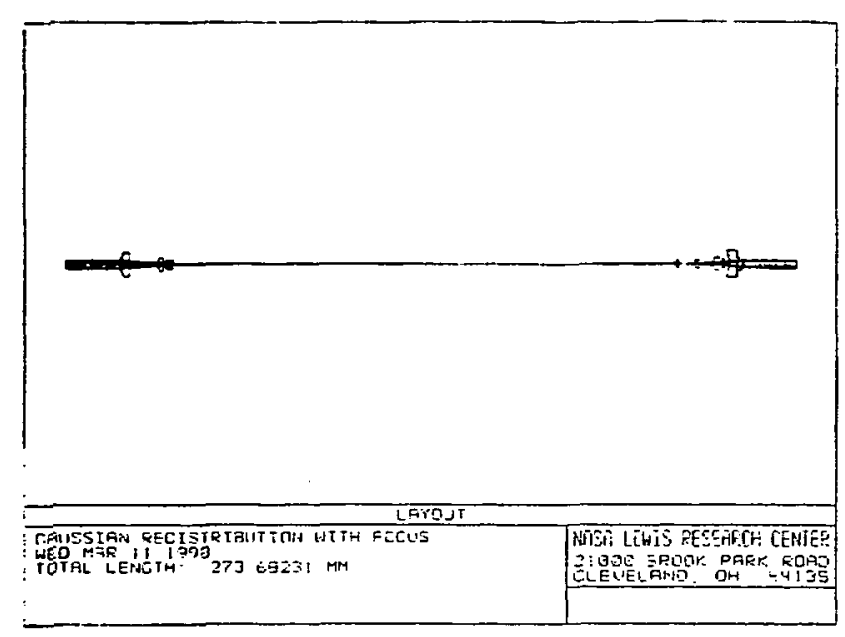

Fig. 1 Layout of focusing system.

first and second elements of the second triplet was required to obtain optimal performance. Figure 1 is a layout of this $(++-,+-+-+)$ focusing system.

An alternate design was found by using a negative lens as the first element with a catalog asphere following it in the first group. This avoided the real focus, which would be undesirable for high-power lasers. In this case the second element of the first triplet was eliminated since it did not contribute to improvement of the system. Additionally, the first element of the second group was split, yielding a design of $(-+,+-+-)$. As mentioned, the first element of the second group is aspheric, but it is a catalog element from Geltech. This system, hereafter referred to as the nonfocusing system, is depicted in Figure 2.

\section{Final Design and System Performance}

In these designs, critical parameters were glass thicknesses and the distance between elements in the first doublet. Preference on the former was always for thinner elements and the position of the latter was the most sensitive parameter in the design of the system which produced a focus.

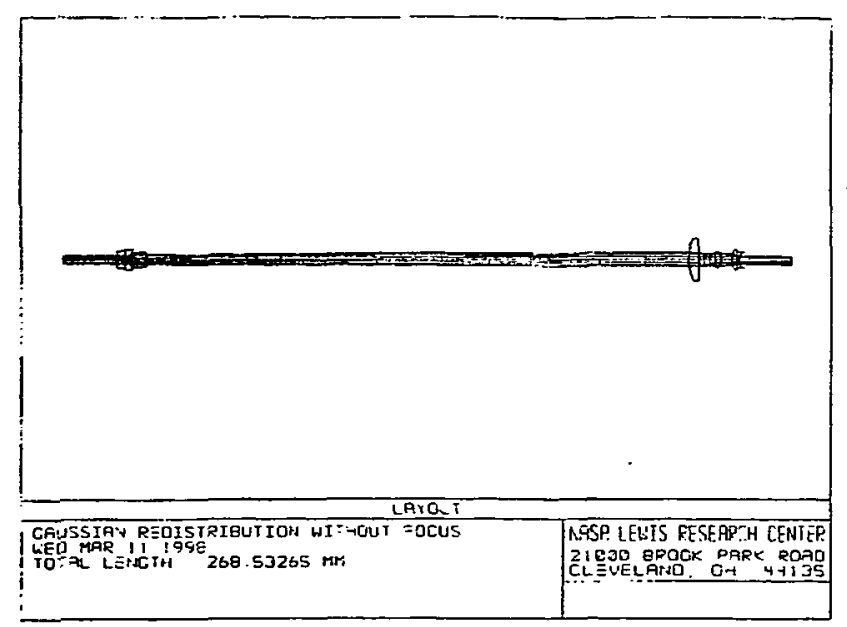

Fig. 2 Layout of nonfocusing system.

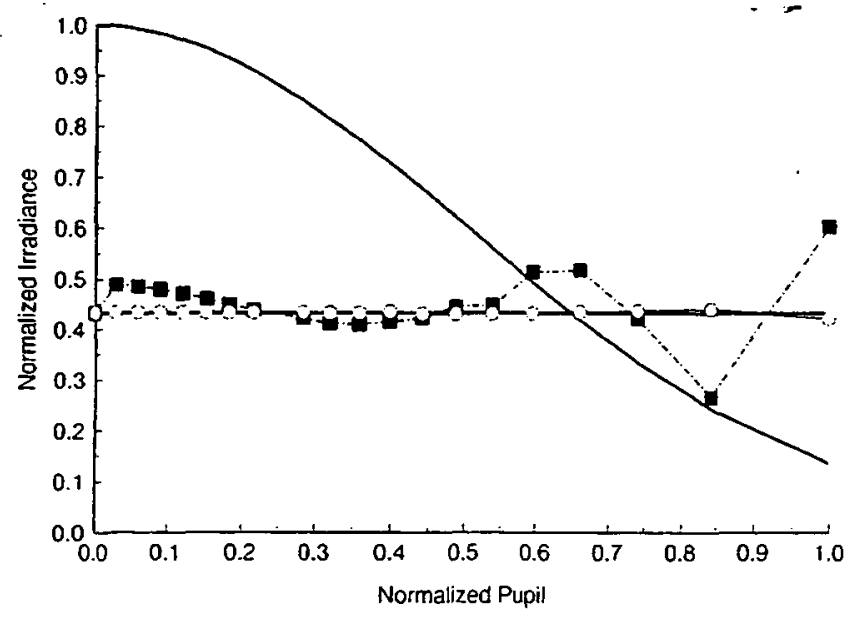

Fig. 3 Irradiance distribution of both systems.

The performance of the two design types is plotted in Figure 3. The desired input Gaussian and output uniform irradiance profiles are plotted for reference. Data points denoted by darkened squares describe the performance of the nonfocusing system whereas the empty circles plot the irradiance for the focusing system. Both of these irradiance distributions were calculated $1 \mathrm{~m}$ from the rear vertex of the last element. As shown in this figure, the focusing system performs much better than the one that did not come to a real focus. The nonfocusing system may be viewed as a local minima in the search for the focusing system since great care was required to prevent the nonfocusing system from producing a focus.

Figure 4 repeats the irradiance plot of Figure 3 for the focusing system but changes the range of the vertical axis to better display the irregularities. Additionally, empty triangles denote the irradiance of this system $2 \mathrm{~m}$ from the rear vertex of the last element. As illustrated, the beam changes very little over this propagation distance. Numerically, the maximum error at both distances is $1.7 \%$ within the $1 / e$ radius and a maximum of $2.6 \%$ at the edge of the field for the $1 \mathrm{~m}$ distance. That error grows to $4 \%$ at $2 \mathrm{~m}$.

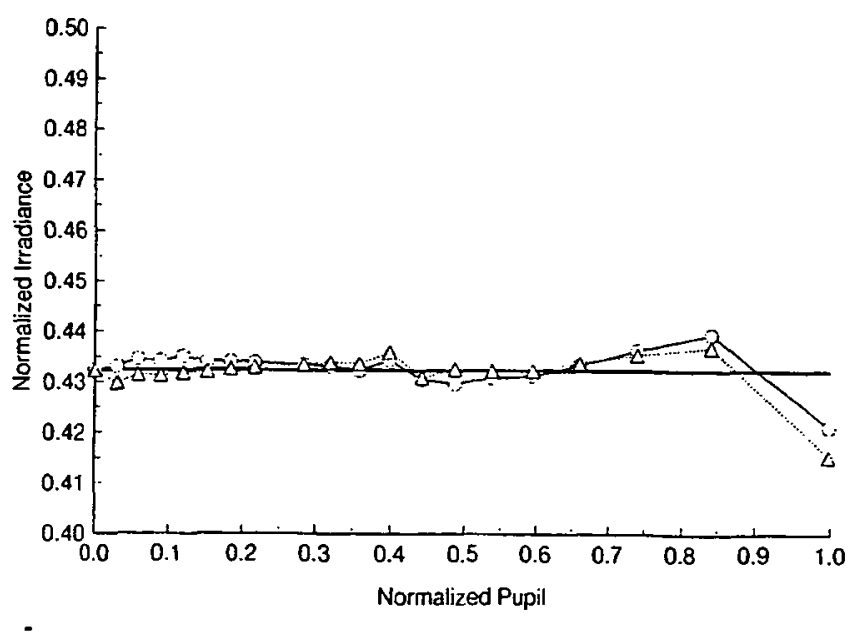

Fig. 4 Irradiance distribution of focusing system at 1 and $2 \mathrm{~m}$. 
Table 1 Prescription for $(++-,+-+-+)$ lens.

\begin{tabular}{rccl}
\hline $\begin{array}{c}\text { Surface } \\
\text { Number }\end{array}$ & Radius & Thickness & $\begin{array}{c}\text { Commercial Part } \\
\text { Number }\end{array}$ \\
\hline 1 & 11.142 & 2.9 & MG LPX 025 \\
2 & & 10.8438 & \\
3 & 5.19006 & 1.9 & OS 011-0230 \\
4 & & 1.97153 & \\
5 & -1.55 & 1.0 & SH 314000 \\
6 & 1.55 & 189 & SH 314000 \\
7 & 1.42999 & 1.5 & JML CPX 10035 \\
8 & & 6.4156 & \\
9 & & 0.5 & \\
11 & 1.55 & 5.83376 & SH 314000 \\
12 & 2.58399 & 2.5 & SH 312007 \\
13 & & 0.67332 & \\
14 & & 0.5 & \\
15 & 1.55 & 2.76431 & SH 314000 \\
16 & -14.3 & 3 & MF LFF088 \\
17 & & 2.38 & ES 45147 \\
18 & -4.65 & & \\
\hline \hline
\end{tabular}

While the design could have retained use of custom radii, catalog elements can greatly reduce the cost while delivering adequate performance, as described elsewhere. ${ }^{7,8}$ Table 1 lists the prescription of the $(++-,+-+-+)$ lens along with the associated manufacturer's part numbers. Similar information is provided in Table 2 for the $(-+$, $+-+-)$ design. In these tables MG refers to Melles Griot, S/H to Spindler and Hoyer, NRC to the Newport Research Corporation, GE to Geltech, ES to Edmund Scientific, and OS to OptoSigma. All distances given are in millimeters and the designs assume a wavelength of $0.6328 \mu \mathrm{m}$.

\section{Conclusion}

The design forms for lens systems that will transform an incident Gaussian beam to an output beam with uniform
Table 2 Prescription for $(-+,+-+-)$ lens.

\begin{tabular}{cccc}
\hline \hline $\begin{array}{c}\text { Surface } \\
\text { Number }\end{array}$ & Radius & Thickness & $\begin{array}{c}\text { Commercial Part } \\
\text { Number }\end{array}$ \\
\hline 1 & -12.92 & 2.5 & NRC KPC 010 \\
2 & & 2 & MG LPK 004 \\
3 & 7.77998 & $0.6997^{-25 i}$ & \\
4 & 6.6276 & 5 & CO 350220 \\
5 & & 200 & \\
6 & 16.962 & 3.9 & MG LPX 057 \\
7 & & 2 & \\
8 & & 2 & MG LPK 002 \\
9 & 5.18699 & 1.98511 & \\
10 & & 2 & SH 312201 \\
11 & -5.464 & 3.93945 & \\
12 & 6.743 & 2 & MG LPX 008 \\
13 & & 0.5 & \\
14 & 3.101 & & SH 314001 \\
\hline \hline
\end{tabular}

intensity over long propagation distances have been presented. The quality of the correction at $1 / e^{2}$ points was presented. Prescriptions for implementation using stock lenses were provided.

\section{References}

1. C. Wang and D. L. Shealy, "Design of gradient-index lens systems for laser beam reshaping," Appl. Opt. 32, 4763-4769 (1993).

2. D. L. Shealy, Private Communication (1993).

3. B. R. Frieden, "Lossless conversion of a plane laser wave to a plane wave of uniform irradiance," Appl. Opt. 4, 1400-1403 (1965).

4. J. L. Kreuzer, "Coherent light optical system yielding an output beam of desired intensity distribution at a desired equiphase surface," U.S. Patent No. 3,476,463 (Nov. 4, 1969).

5. C. Y. Han, Y. Ishi, and K. Murata, "Reshaping collimated laser beams with Gaussian profile to uniform profiles," Appl. Opt. 22, 3644-3647 (1983).

6. D. Shafer, "Gaussian to flat-top intensity distributing lens," Optics and Laser Technology, 159-160 (1982).

7. D. R. Buchele and D. W. Griffin, "Compact color Schlieren optical system," Appl. Opt. 32, 4218-4222 (1993).

8. J. M. Sasian, F. B. McCormick, R. Webb, R. J. Crisci, K. O. Mersereau, and R. P. Stawicki, "Design, assembly and testing of an objective lens for a free-space photonic switching system," Opt. Eng. 32, 1871-1877 (1993). 